\title{
Engineered T cells Flt around their targets
}

Professor Emma C Morris, UCL, London

Email: e.morris@ucl.ac.uk

Antigen escape by solid tumors have limited the efficacy of genetically modified T cells. T cells engineered to secrete the cytokine Flt3L induce activation of endogenous $\mathrm{T}$ cells targeting a broader repertoire of tumor antigens via the expansion of intratumoral antigen presenting cells, significantly improving tumor responses.

The recent therapeutic impact of genetically modified T cells on some forms of cancer, particularly B cell lymphomas, has been profound ${ }^{1}$. As a result, a paradigm shift in treating hematological malignancies has occurred. But these results have not been recapitulated in the more common solid tumors. A major factor impacting the response to immune therapies is the heterogenous expression of tumor antigens within individual tumor masses, between primary tumours and distant metastases and also changes in antigen expression over time ${ }^{2}$. In these instances, single specificity $T$ cell therapies are unable to recognise and kill all malignant cells and tumor eradication only rarely occurs. In this issue of Nature Immunology, Lai et al. have demonstrated that by engineering the adoptively transferred tumour antigenspecific T cells to also secrete the dendritic cell (DC) growth factor Flt3L, the infused T cells can activate professional antigen presenting cells (specifically, $\mathrm{CDC1s}$ ) within the tumor in addition to directly recognising antigen positive tumor cells $^{3}$. Once activated, the intratumoral dendritic cells expand and stimulate endogenous (host) T cells within the tumor microenvironment. The activated polyclonal endogenous $T$ cells are able to recognise a broad range of tumorassociated antigens, more effectively targeting heterogenous tumors. Chimeric antigen receptor (CAR) and T cell receptor (TCR) engineered T cells further modified to secrete Ft3L generated superior tumor immunity through the simultaneous delivery of potent mono-specific $T$ cells and the induction of host immune responses directed against multiple tumor antigens, commonly referred to as epitope spreading ${ }^{4}$.

As a physician, I don't like the concept of 'fighting cancer'. The implication that for patients dying of the disease it could somehow have been their fault, that they didn't try hard enough, compounds fear and grief with inappropriate shame. But at a cellular level it is, absolutely, a fight to the death. Harnessing the power of the innate and adaptive immune system to eradicate cancer is no longer fantasy. Continuing the 'fight' analogy, arming T cells through genetic engineering has equipped them with an array of weapons to enhance (i) recognition of tumor cells, through the expression of tumor-specific receptors such as CAR and TCR; (ii) effector function of the infused T cells; and (iii) persistence in vivo. However, despite remarkable success in the treatment of various B cell malignancies achieving genuine, durable cures where all other therapies had failed, the tumors are fighting back. This is not surprising.

Complex, dynamic interactions exist between tumor cells, the tumor microenvironment and the host immune response. Tumor-associated antigens and neo-antigens expressed by tumor cells stimulate an anti-tumor immune response, subjecting the tumor to immune editing by various mechanisms ${ }^{5}$. These include selection for DNA copy number loss of clonal neoantigens, transcriptional repression of neoantigens, and loss or mutation of human leukocyte antigen (HLA) and antigen-presentation components, amongst others. Intrinsic genetic heterogeneity of the tumor also impacts on disease progression and susceptibility to tumor directed immunity. Detailed TCR repertoire analysis of tumor infiltrating T cells can be used to assess the breadth and strength of the T cell response ${ }^{6}$. Tumors with a high mutational load (i.e. express a greater number of neo-antigens) are associated with reduced disease recurrence after checkpoint inhibitor therapy such as pembrolizimab (anti-PD1), mediated by a polyclonal repertoire of non-tolerant, neoantigen-specific, self-restricted endogenous T cells ${ }^{7}$. 
Elsewhere, the first CAR- and TCR-T cells used clinically have targeted single, immunodominant tumor antigens, such as CD19, CD20, BCMA, NyEso and WT1 (the latter two as peptide epitopes presented by Class I MHC). Clinical data for CD19 CAR T cells now has up to 8 years follow-up in the earliest recipients. When relapse occurs, it is frequently CD19 negative in patients with $B-A L L^{8,9}$, which has also been observed following other CD19-targeted immunotherapies, such as blinatumomab. In the context of B cell solid tumors, such as DLBCL, sequential loss of tumor antigens following CAR T-cell therapies has been observed ${ }^{10}$. These cases highlight the importance of immune-mediated selective pressure driving tumor evolution with loss of antigen expression. New strategies are therefore urgently required to mitigate against tumor escape and tumor heterogeneity following highly potent monospecific T cell therapies. Some approaches described to date include the use of tandem CARs, dual CARs, multispecific CARs, co-administration of CAR T cells and co-transduction of T cells with multiple CARs, all comprehensively discussed in recent reviews ${ }^{11,12}$.

With the overall aim of demonstrating that soluble Flt3L delivered to the tumor site can activate DCs and enhance the activation of endogenous tumor infiltrating $T$ cells resulting in improved anti-tumor immunity a number of elegant experiments have been performed by Lai et $\mathrm{al}^{3}$. The authors first demonstrate that modifying tumor cell lines to overexpress soluble Flt3L effectively drive the expansion of intratumoral $C D 103^{+} C D C 1 s$, a subset of dendritic cells important for cross-priming and activation of anti-tumor $\mathrm{CD} 8^{+} \mathrm{T}$ cells and increase the frequency of intratumoral $\mathrm{CD} 8^{+} \mathrm{T}$ cells.

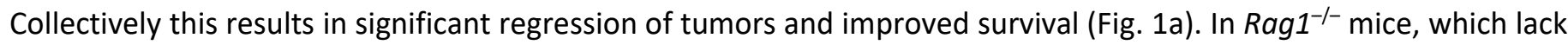
$\mathrm{T}$ cells (and B cells), the presence of Flt3L had no effect, highlighting the dependence on endogenous T cells for tumor control. Based on these observations, Lai et al. proceed to engineer tumour antigen-specific T cells (TCR transgenic and CAR expressing) to secrete Flt3L as a novel approach to enhance Flt3L concentration at the tumor site and promote anti-tumor immunity. Bone marrow-derived DCs cultured in the presence of supernatant harvested from T cells stably transduced to secrete Flt3L underwent significantly increased expansion and differentiation into CD103 ${ }^{+}$DCs (Fig. 1b), supporting enhanced stimulation of antigen-specific $T$ cells as measured by production of the inflammatory cytokines IFN- $\gamma$ and TNF. The antigen-specific cytokine secretion was further enhanced in the presence of other adjuvants, such as polyl:C.

Three murine models were employed to test the anti-tumor efficacy of Flt3L-secreting T cells. In the first, OT1 TCR transgenic T cells were transferred into tumor bearing mice. All OT1 T cells express the same $\alpha \beta-T C R$, which recognises the OVA peptide processed and presented by MHC class I molecules $\left(\mathrm{H}_{2} \mathrm{~K}^{\mathrm{b}}\right)$. In this model, full activation of the OT1 T cells requires cognate antigen (OVA) presentation by DCs. The other two models tested the additive effect of Flt3L secretion on the anti-tumor function of anti-Her2 CAR T cells in mice bearing either Her2-expressing colon adenocarcinoma or Her2-expressing breast cancer. Again, the potent in vivo anti-tumor effects observed could be augmented further by combination with immune agonists (Fig. $1 \mathrm{c}$ and d). In support of the therapeutic effect being dependent on the Flt3L and $\mathrm{CD}_{103^{+}} \mathrm{DC}$ mediated recruitment and activation of endogenous (host) T cells, TCR repertoire analysis was performed confirming the oligoclonal expansion of host $\mathrm{T}$ cells, identified in the tumor-draining lymph nodes. Strikingly, the authors were able to demonstrate that Flt3L secreting anti-Her2 CAR T cells in combination with immune adjuvants were able to induce host $T$ cell responses towards non-CAR antigens, delineated through the use of tumor cells co-expressing Her2 (the CAR target) and OVA (an immunogenic 'model' tumor antigen), with clear emergence of OVA-reactive endogenous host $T$ cells at the tumor site, resulting in a superior anti-tumor effect. Durability of anti-tumor responses is critical for clinical benefit. To this end, Lai et al provide convincing data from a secondary re-challenge model using a tumor cell line lacking the CAR and/or TCR target antigens that treatment with Flt3L-secreting CAR T cells and immunological adjuvants can generate long-term host memory antitumor responses towards tumor-associated antigens beyond the CAR or TCR target.

The ability to stimulate a broad and potent immune response towards multiple tumor antigens, through the activation of the endogenous $\mathrm{T}$ cell repertoire offers a genuine solution to the limitations inherent in targeting tumor antigen heterogeneity in solid tumors with monospecific $\mathrm{T}$ cell therapies. Back to the fight. Flt3L-secreting anti-tumor T cells may just have the upper hand. By recruiting other immune effectors to their cause, a sustained attack on more than one front is now possible. As a believer in teamwork, this is not surprising. 


\section{Competing interests}

The authors declare no competing interests

\section{Schematic representation of anti-tumor immunity generated by adoptively transferred gene-modified T cells (CAR-T or TCR-T) with or without additional modification to secrete FLt3L.}

(a) Endogenous T cells are typically unable to prevent tumor growth due to multiple factors including the immunosuppressive microenvironment, tolerance to self and T cell exhaustion.

(b) Following the adoptive transfer of gene-engineered tumor antigen-specific T cells (eg CAR-T or TCR-T), antigen expressing tumor cells are recognised directly, in the case of CAR T cells or via presentation of peptide/MHC complexes to TCR-T cells (not shown) resulting in killing of antigen-expressing tumor cells as long as the transferred anti-tumor $\mathrm{T}$ cells persist and remain functional.

(c) In the face of immune pressure and due to inherent genetic instability, tumor progression (escape) can occur through various mechanisms including (i) antigen loss or (ii) clonal evolution. Both scenarios result in loss of response to transferred single antigen-specific engineered T cells. In the case of clonal evolution, tumor heterogeneity results in the expression of neo-antigens.

(d) The transfer of tumor antigen-specific T cells engineered to also secrete Flt3L generates high concentrations of soluble Flt3L at the tumor site. This results in the additional activation and expansion $\mathrm{CD}_{103^{+}}$dendritic cells, which can activate and recruit endogenous polyclonal T cells, able to recognise neo-antigens and non-CAR target antigens. This phenomenon of epitope spreading was enhance by the presence of immune adjuvants, such as poly I:C.

\section{References}

1. Brown CE, Mackall CL. Nat Rev Immunol. 2019;19(2):73-74.

2. Jamal-Hanjani M, et al. N Engl J Med. 2017;376(22):2109-2121.

3. Lai J, et al. Nat Imm 2020

4. Vanderlugt $\mathrm{CL}$, Miller SD. Nat Rev Immunol. 2002;2(2):85-95.

5. Schreiber RD, et al. Science. 2011;331(6024):1565-1570.

6. Joshi K, et al. Nat Med. 2019;25(10):1549-1559.

7. Gubin MM, et al. Nature. 2014;515(7528):577-581.

8. Maude SL, et al. N Engl J Med. 2018;378(5):439-448.

9. Park JH, et al. N Engl J Med. 2018;378(5):449-459.

10. Shalabi H, et al. Haematologica. 2018;103(5):e215-e218.

11. Majzner RG, Mackall CL. Cancer Discov. 2018;8(10):1219-1226.

12. Rafiq S, et al. Nat Rev Clin Oncol. 2020;17(3):147-167. 